Georgetown University Institutional Repository http://www.library.georgetown.edu/digitalgeorgetown

The author made this article openly available online. Please tell us how this access affects you. Your story matters.

Carlson, K. A., Meloy, M. G., \& Russo, J. (2006). Leader-Driven Primacy: Using Attribute Order to Affect Consumer Choice. Journal Of Consumer Research, 32(4), 513-518. doi: $\underline{10.1086 / 500481}$

Collection Permanent Link: hdl.handle.net/10822/707943

(C) 2006 University of Chicago Press.

This material is made available online with the permission of the author, and in accordance with publisher policies. No further reproduction or distribution of this copy is permitted by electronic transmission or any other means. 


\title{
Leader-Driven Primacy: Using Attribute Order to Affect Consumer Choice
}

\author{
KURT A. CARLSON \\ MARGARET G. MELOY \\ J. EDWARD RUSSO*
}

\begin{abstract}
Leader-driven primacy uses initial product information to install a targeted brand as the early leader in a choice between two brands. Biased evaluation of subsequent attributes builds support for the targeted brand, causing the choice itself to be biased. Study 1 finds evidence of this effect in choices between two equally attractive brands. Study 2 extends the finding to a situation where one brand is inferior and to conditions where participants do not explicitly identify their leader. Study 3 shows how leader-driven primacy can be reduced by encouraging brandbased processing.
\end{abstract}

C onsumers often construct new preferences to support existing ones. Though this tendency was once thought confined to strongly held preferences, it also occurs when consumers only slightly prefer one brand over another. Throughout this article, we refer to the brand that has this early advantage in preference as the leading brand. A growing body of research reveals that a leading brand naturally emerges early in most choices and that consumers tend to construct preferences to support that leader (Brownstein 2003). What has not been established is whether biased predecisional processing to support a leader has a nonnormative influence on choice.

This issue is open in part because experiments have generally confounded leadership and true preference. Since leaders emerge naturally from unbiased preferences over early information, brands that gain the initial lead are often genuinely superior and so should be selected. To establish whether leadership has a nonnormative impact on choice, the effect of true preferences must be removed. The present work uses information order to do so. By manipulating which of two brands in a binary choice is favored by the first attribute, either brand can be installed as the initial leader. Because all participants eventually see the exact same product information, a difference in brand choices across

*Kurt A. Carlson is assistant professor, Fuqua School of Business, Duke University, Durham, NC 27707 (kurt.carlson@duke.edu). Margaret G. Meloy is assistant professor, Smeal College of Business, Pennsylvania State University, University Park, PA 16802 (mmeloy@psu.edu). J. Edward Russo is professor, Johnson Graduate School of Management, Cornell University, Ithaca, NY 14853 (jer9@ cornell.edu). Correspondence should be addressed to Kurt Carlson. The authors thank Joel Huber, Hans Baumgartner, and three reviewers for helpful comments on earlier drafts of this article. information orders cannot be due to differences in true preferences. This article examines the biased construction of attribute evaluations to support an installed leader and the resulting overselection of that leader. We refer to this process phenomenon as leader-driven primacy.

To see how consumers might construct attribute-level preferences to support early leaders, consider the case of a student who is deciding between two backpacks described by several attributes, such as the following.

Backpack $\mathbf{A}$ has a bottom that is double-stitched and double-lined for durability. In addition, the material has been treated to enhance its strength and suppleness.

Backpack B has a layer of leather sewn into the bottom of the pack to enhance its integrity. The leather has been treated with oil for a softer, richer feel.

If the student would normally evaluate this attribute as neutral when seeing it in isolation, then a leader-supporting bias would appear as a shifting of its evaluation to favor backpack A (B) when backpack A (B) is leading.

This type of proleader biasing of attribute evaluations has been documented in several consumer choice studies (e.g., Carlson and Pearo 2004; Russo, Meloy, and Medvec 1998). It is similar to selective hypothesis testing (e.g., Hoch and Ha 1986; Posavac et al. 2004), where the leading option is the pet hypothesis, and biased predecisional processing is the method of selective testing that supports it. However, it is a nontraditional form of selective hypothesis testing because it does not depend on either selective recruitment of information from memory or from the environment but instead on biased attribute evaluations. Among the ways that 
consumers can support their leaders during preference construction, biased construction of attribute evaluations is arguably the most general because it can be used by anyone on any information, regardless of how the information was acquired.

Recent work on predecisional processing suggests that individuals may bias their attribute evaluations to achieve a metacognitive consistency goal (Carlson and Russo 2001; Simon, Krawczyk, and Holyoak 2004). For example, Simon et al. (2004) contend that consumers' fundamental desire to align the mental representations of the choice options leads them to bias their attribute evaluations toward interattribute consistency. The ultimate consequence of this leader-supporting bias is an exaggerated separation in the overall values of the options.

As noted above, we know of no evidence indicating that early leaders bias brand choice. Possibly this lack of evidence occurs because consumers support their leaders only to bolster confidence and stop short of sacrificing choice accuracy. Then, whenever new information suggests that the trailing option is superior, they correctly switch the leader. Indeed, both Carlson and Russo (2001) and Russo et al. (1998) found that half of all decision makers switched leaders at least once during a binary choice process.

In spite of the evidence that leadership reversals are common and the compelling logic that the order of information should not affect the option chosen, we expect that early leadership will materially impact brand choice. We reason that placing an attribute in the first serial position that clearly favors one of the two brands (hereafter referred to as the targeted brand) will induce most individuals to adopt that targeted brand as their early leader. Then, consumers will bias their evaluations of new attributes to support the leader and eventually overselect it. To test this prediction, we manipulate which attribute appears first, thereby controlling which brand becomes the leader. Despite the simplicity of this manipulation, we are not aware of any study that has explored the impact of such a manipulation on consumer choice.' Just as important as the demonstration of such an order effect is the identification of its causal underlying mechanism. To this end, we collect predecisional attribute evaluations so that we can determine whether attribute-level preferences tend to be constructed to favor the installed leader.

\section{STUDY 1: CHOICE BETWEEN EQUALLY ATTRACTIVE BRANDS}

\section{Method}

Stimuli. All participants made two choices, one between two backpacks, the other between two restaurants. Each

\footnotetext{
'Studies that have explored other order effects on choice have focused on order of alternatives (Kardes and Kalyanaram 1992), of choice tasks (Drolet 2002), and of the comparisons between the options (Dhar and Simonson 1992; Mantel and Kardes 1999). However, none of these studies has explored the influence of the order of product information on brand choice.
}

choice was motivated by a brief scenario, such as buying a backpack for a younger sibling who was going to college.

The two brands in each choice were described by six attributes, such as the one shown above. For backpacks, the attribute categories were construction, dimensions, history of the company, materials, prices, and zippers. For restaurants, they were ambience, desserts, dining-guide ratings, location, menu, and parking. Four of the attributes in each domain were neutral in that they were written, pretested, and rewritten until they favored neither brand (e.g., see the attribute above). When evaluated by pretest participants on a nine-point evaluation scale anchored at either end by the two options, these attributes' average value was near 5. In addition, the standard deviation of these evaluations typically centered on 1.5 , indicating substantial differences among participants' evaluations. Thus, a particular participant might consider any of these attributes to favor one brand or the other, but across participants, these attributes favored neither brand (and were uncorrelated with each other).

The two remaining attributes in each domain were designed to be diagnostic in opposite directions. That is, each of these attributes favored one of the brands by a small but relatively clear amount, such as the desserts attribute below that favors restaurant B.

Restaurant A has a small assortment of standard desserts, but daily specials always feature one type of cheesecake, a fruit sorbet, and a chocolate dessert.

Restaurant B has a variety of desserts including cakes, tortes, pies, sorbet, and ice cream. In addition, specialty desserts are prepared daily. Dessert and coffee are included in the price of the meal. A professional dessert chef is on staff and will accommodate requests.

When evaluated by pretest participants, these attributes had mean evaluations that were reliably above (6.5) or below (3.5) neutral, with roughly half of the variability of the four equivocal attributes.

Design. The focal manipulation was the placement of one or the other of the diagnostic attributes as the first information participants saw. The opposing brand's diagnostic (i.e., targeting) attribute was put in the fourth serial position, and the remaining (neutral) attributes were randomly assigned to the second, third, fifth, and sixth positions. The brand that was targeted, the order of the four neutral attributes, and the order of the two choices (backpacks and restaurants) were all counterbalanced. Neither the order of the neutral attributes nor the order of the choice domains yielded any significant differences, so they are not discussed further.

Participants and Procedure. Fifty-four undergraduate students participated in exchange for extra course credit. Three decision progress questions were used after each attribute to determine whether participants biased their attribute evaluations during the choice process. The first question asked participants, "Please consider the information that you 
have just received. Rate it on the scale below according to your personal judgment." Responses were recorded on the nine-point evaluation scale described above. The second and third questions measured which brand was leading up to that point in the choice process and how confident participants were in their leader. After evaluating all six attributes, participants selected their preferred brand.

\section{Results}

Installing the Leader. In both choice domains, 51 of 54 participants (.94) indicated that the targeted brand was leading after the first attribute. Thus, the installation of the targeted brand as the initial leader was largely successful.

Biased Choice. Our primary research question was whether arbitrary installation of one brand as the initial leader would bias choice above chance (.50). The targeted backpack was chosen by 36 of the 54 participants (.67) and the targeted restaurant by 40 of $54(.74)$. A Gaussian approximation to the binomial distribution revealed that both proportions were significantly above chance (restaurants: $z=3.54, p<.001$; backpacks: $z=2.45, p<.01$ ). Combining across product domains, the mean choice proportion of the targeted brand was 0.70 . Thus, targeting and the leadership it created had a clear, reliable influence on choice.

Biased Attribute Evaluations. The next research question was whether the cause of the choice effect was a bias in the attribute evaluations to support the installed leader. To answer this question, we calculated each participant's average attribute evaluation over the neutral attributes (i.e., those appearing in positions 2, 3, 5, and 6) and compared the mean of these average evaluations across targeting conditions. This aggregation was deemed appropriate because comparisons among the four neutral attributes for each targeting condition revealed no differences between any pair (all $p>.15$ ). Since a rating of 5 represented indifference on the nine-point evaluation scale, evaluations below (above) this value favored brand $A(B)$.

In the restaurant choice, the mean attribute evaluation of the four neutral attributes was lower (4.28) when brand A was targeted than when brand B was targeted (6.39). Both means differed from neutrality in the predicted direction $(t(26)=2.19$ for $\mathrm{A}$ and $t(26)=4.83$ for $\mathrm{B}$; both $p<.05)$. Similarly, for backpacks, the average evaluation of the neutral attributes was 3.67 when brand A was targeted and 5.67 when brand B was targeted. Again, both means were significantly different from neutrality (both $p<.01$ ). In sum, even though the attributes in positions $2,3,5$, and 6 were pretested to be neutral, participants saw them as diagnostic in favor of the brand that had been targeted to become the early leader by the first attribute.

Finally, we examined whether participants biased their evaluations of the fourth attribute, which objectively favored the nontargeted brand. For economy of exposition, we combined the two product domains and the two targeting attributes for each domain. The mean evaluations of the four attributes were 1.84 units from 5 in the appropriate direction when they appeared in the first serial position and 0.17 in the opposite direction when in the fourth position. The difference was significant $(p<.05)$, a result that held for all four attributes tested individually (all $p<.05$ ). Thus, even the diagnostic attributes were consistently distorted toward the targeted brand.

\section{Discussion}

This study shows that it is possible to target a brand in a binary choice by putting information favorable to it in the initial position. Because the targeted brand generally became the early leader, biased attribute evaluations to support the leader resulted in a leader-driven primacy effect, with $70 \%$ of participants selecting the targeted brand. These data provide initial evidence that leader-driven primacy can occur in consumer choice and that it derives from predecisional restructuring of attribute evaluations.

An alternative account that cannot be eliminated by the current study is that individuals presumed that attribute order reflected the importance of the information provided, with the most important information appearing first (Hogarth and Einhorn 1992). If so, then attribute importance weights collected after the choice process should reflect this. To test this competing explanation, we replicated study 1 with a new choice domain (resort hotels) and a new sample of 260 participants. After seeing the hotel attributes in one of two orders (targeting one hotel or the other) and making their choice, participants allocated 100 importance points across the six attributes.

All main results of the previous study were replicated. The proportion of participants who selected the targeted hotel was greater than chance $(.63 ; z=4.32, p<.001)$, and both the neutral and targeting attributes exhibited the appropriate differences across targeting conditions (both $p<$ .01). Next, we tested whether importance weights for the two targeting attributes were higher when they appeared first than when they appeared in the fourth position. This test yielded no difference in importance weights for either targeting attribute (both $p>.25$ ). Thus, individuals did not infer importance of the targeting attribute from attribute order. This is not to say that consumers do not alter attribute importance weights to achieve a coherent representation of the choice options; evidence suggests that they do (e.g., Simon et al. 2004). Instead, these data show that leaderdriven primacy does not require biased attribute weighting.

The possibility exists that the choice result above was due partly to individuals explicitly updating their preference after each attribute and determining their current leader. If so, our results might have been driven by the choice progress questions we asked. A second possibility is that our results depend in part on the parity in brand attractiveness. Maybe most participants would have been satisfied with either brand, so the choice effect is of little consequence. This raises the question of whether leader-driven primacy generalizes to a choice between brands that are not equally 
attractive to most participants. These two issues were addressed in study 2 .

\section{STUDY 2: CHOICE OF AN INFERIOR BRAND}

The first study was designed to be highly sensitive to the process underlying leader-driven primacy. The nontargeting attributes were neutral, on average, in order to provide the maximal opportunity for bias in attribute evaluations (Russo et al. 1998), and the choice process was closely tracked by asking the three progress questions after every attribute. This study involves a greater hurdle for leader-driven primacy to overcome. First, the nontargeting attributes are not neutral but instead slightly favor one of the brands. This should reduce the room for bias. Second, to determine whether leader-driven primacy depends on asking the progress questions, we include a condition in which those questions are omitted.

\section{Method}

In exchange for course extra credit, 230 undergraduate students participated in this study, which mirrored study 1 with three exceptions. First, some participants answered the progress questions after each attribute, while others did not. Second, the choice domain was winter coats. Third, the attribute information was written and pretested so that brand $\mathrm{B}$ was more attractive (on average) than brand $\mathrm{A}$. This was accomplished by writing the four nontargeting attributes to slightly favor brand B. Though five of the six attributes favored brand B (all but the targeting attribute), there was sufficient ambiguity in the four nontargeting attributes that consumers could pick brand $\mathrm{A}$ and feel capable of defending their choice to a dispassionate observer.

The design was a 2 (target: brand A, brand B) $\times 2$ (questions: present, not present) between-participants design. Participants used six attributes to select a winter coat. Each brand was targeted for half the participants by presenting a protarget attribute in the first serial position and the competitor's targeting attribute in the fourth serial position. Those in the standard questions-present condition answered the three-choice progress questions after each attribute, while those in the condition without questions did not.

\section{Results}

A majority of participants (.77) selected brand B over brand $\mathrm{A}$, verifying that it was superior on average. However, our main research questions were whether leader-driven primacy could induce the choice of an inferior brand (brand A) and how much of this effect would remain when the progress questions were omitted. To jointly assess the impact of targeting and questions, choice of the superior coat was regressed (via a binary logistic regression) on these two factors and an interaction term. Results revealed no interaction effect $(z=.33, p>.50)$ and no main effect of the questions factor $(z=.54, p>.50)$, indicating that the effect of targeting on choice did not depend on the presence of the progress questions. However, as expected, a significant main effect of targeting emerged $(z=2.79, p<.01)$.

The more attractive coat (brand B) was selected more often when it was targeted (.84) than when it was not $(.68$; $z=2.88, p<.01$ ). Thus, even when one brand was preferred three-to-one, targeting influenced choice. The difference in choice due to targeting was $16 \%$ for the questionspresent conditions (.86 vs. $.70 ; z=2.43, p<.05)$ and $15 \%$ for the questions-not-present condition (.80 versus .65 ; $z=1.52, p=.13$ ). The attribute evaluation data replicated those from study 1 . The four nontargeting attributes were evaluated as more favorable to brand $\mathrm{B}$ when it was targeted $(M=6.13)$ than when brand A was targeted (5.53; $t(148)=2.49, p<.05)$, and the two targeting attributes were seen as more diagnostic when they appeared in the first than in the fourth serial position (both $p<.05$ ).

\section{Discussion}

This study shows that leader-driven primacy generalizes to choices where the brands differ in overall attractiveness and that leader-driven primacy does not depend on consumers' explicitly updating their leader or reporting their attribute evaluations. One way to view these results is by choice of the inferior coat. When it was not targeted, its choice proportion was .16 , but when it was targeted, its choice proportion rose to .30 . This $14 \%$ difference represents individuals who, because of leader-driven primacy, selected an inferior coat.

A remaining challenge is how to reduce leader-driven primacy. This task is complicated by the failure of some obvious tactics. For starters, leaders form naturally during most choice processes, so it may be impossible to eliminate leader-driven primacy by suppressing their emergence. Even prospective jurors in a courthouse who were instructed to avoid coming to a premature conclusion formed an early leader and biased their evaluation of new information to favor it (Carlson and Russo 2001). In addition, the tendency to bias information evaluations to favor one's leader is remarkably robust (Brownstein 2003) and would seem to be equally difficult to suppress. Because of the difficulty of inhibiting the emergence of a leader or preventing the biased evaluation of information, we sought another approach.

\section{STUDY 3: DISCOURAGING ATTRIBUTE- BASED PROCESSING}

We reasoned that leader-driven primacy might be reduced if consumers could be discouraged from using attributebased processing. Specifically, if brand comparison within attributes could be inhibited, leader-driven primacy might be reduced.

Since previous research has found that attribute-based processing declines when brands are hard to compare by attribute (Chakravarti and Janiszewski 2004; Johnson 1989), we designed an information format that would make byattribute comparisons difficult. We presented all the infor- 
mation for one brand on a single page and the corresponding information for the other brand on a second page. However, a leader had to be initially installed on its own separate page (as in the studies above). Otherwise, we would not know which brand was the leader and so could not assess leaderdriven primacy. Thus, the information format consisted of three pages: one with the first attribute to install a leader, one with all of the remaining attributes for brand $\mathrm{A}$, and one with all of the remaining attributes for brand B.

\section{Method}

This study was a slightly modified replication of study 1 , where the choice options were two brands of restaurants that were equally attractive on average. As in study 1 , there were two types of attributes, four neutral ones and two diagnostic, which were used for targeting. Participants were assigned either to the same attribute-based format of studies 1 and 2 or to the new information display designed to inhibit attributebased processing. Thus, the design was a 2 (target : brand A, brand B) $\times 2$ (processing format: attribute-based, brandbased) between-participants design.

Participants $(n=84)$ in the attribute-based processing condition read the attributes in sequence and answered the three progress questions after each attribute, just as in studies 1 and 2. The progress questions could not be presented in the brand-based condition.

\section{Results}

To assess the impact of targeting and format on choice, a binary indicator of choice was regressed (via a binary logistic regression) on these two factors and an interaction term. The estimation revealed a significant interaction effect $(z=2.61, p<.05)$. Targeting had a reliable impact on choice for those in the standard (attribute-based) condition $(z=6.29, p<.001)$ but not for those in the brand-based condition $(z=1.19, p>.20)$. The essential result is the proportion of participants who chose the targeted brand in the two conditions. These proportions were .86 for the attribute-based group and .58 for the brand-based group. The former was significantly greater than chance $(z=4.33$, $p<.001)$ and replicated the results of study 1 , while the latter was not reliably above chance $(z=1.15, p>.20)$. Thus, brand-based processing succeeded in reducing leaderdriven primacy.

As in the attribute-based studies above, the nontargeting or neutral attributes were evaluated as supportive of whichever brand was initially targeted $(M=3.13$ when brand A was targeted and $M=6.70$ when brand B was targeted). Both values were significantly different from neutral (both $p<.001$ ). Similarly, the targeting attributes were more strongly valenced when they appeared first than when they appeared fourth (both $p<.05$ ).

\section{GENERAL DISCUSSION}

Unlike postdecisional bias to support a chosen option, which cannot affect the alternative that is chosen, the possibility has always existed that biased predecisional processing impacts choice accuracy. However, the possibility has also existed that biased predecisional processing serves only to harmlessly bolster choice confidence. To the best of our knowledge, the studies above provide the first causal evidence that a leading option, and the biased attribute evaluations that support it, have material consequence for choice accuracy.

Leader-driven primacy has wide implications for consumer decision making. The ability to target a brand or product through information order establishes the possibility of an adversary using information sequencing to influence consumer choice. For example, salespeople can use information order to install a self-serving leader via direct-toconsumer sales pitches, online information displays, and comparative advertising, inter alia. Marketers have the substantial advantage that consumers seem unaware that they are biasing their own evaluation of new information to support whichever brand is leading. In addition to information order, there are several other tactics that might be used to install a particular brand as the early leader. For instance, either mere exposure to a brand or a humorous ad might create a positive initial disposition toward the sponsoring brand.

Leader-driven primacy might well occur in situations beyond binary choice. In principle, it should generalize to a consideration set that contains more than two brands. So long as a leading brand emerges, the evaluation of new attributes should tend to be biased to support it. It should also generalize to yes-no purchase decisions involving a single brand. Finally, although we focused on consumers' preferential choices, leaders are likely to emerge and influence choice in other domains as well. The tendency to evaluate new information to support one's leader has been found in decisions made by prospective jurors (Carlson and Russo 2001), managers (Russo, Meloy, and Wilks 2000), and students choosing a career (Simon et al. 2004). Thus, it is likely that leader-driven primacy exists in nonpreferential domains as well.

In the nearly $40 \mathrm{yr}$. since researchers proposed the idea of a predecisional leading option, a plethora of research has explored how the emergence of an early leader influences predecisional processing. The main conclusions from this body of work are: $(a)$ leaders emerge naturally during choice processes, $(b)$ decision makers tend to support their leaders through biased predecisional processing, and $(c)$ the foregoing have implications for how confident decision makers are in their choices. To these conclusions, we can now add the result that leaders materially influence which option is selected, an effect we have termed leader-driven primacy.

[Dawn Iacobucci served as editor and Eugene Anderson served as associate editor for this article.] 


\section{REFERENCES}

Brownstein, Aaron L. (2003), "Biased Predecisional Processing," Psychological Bulletin, 129 (4), 545-68.

Carlson, Kurt A. and Lisa Klein Pearo (2004), "Limiting Predecisional Distortion by Prior Valuation of Attribute Components," Organizational Behavior and Human Decision Processes, 94 (1), 48-59.

Carlson, Kurt A. and J. Edward Russo (2001), "Biased Interpretation of Evidence by Mock Jurors," Journal of Experimental Psychology: Applied, 7 (2), 91-103.

Chakravarti, Amitav and Chris Janiszewski (2004), "The Influence of Generic Advertising on Brand Preferences," Journal of Consumer Research, 30 (March), 487-502.

Dhar, Ravi and Itamar Simonson (1992), "The Effect of the Focus of Comparison on Consumer Preferences," Journal of Marketing Research, 29 (November), 430-40.

Drolet, Aimee (2002), "Inherent Rule Variability in Consumer Choice: Changing Rules for Change's Sake," Journal of Consumer Research, 29 (December), 293-305.

Hoch, Stephen J. and Young-Won Ha (1986), "Consumer Learning: Advertising and the Ambiguity of Product Experience," Journal of Consumer Research, 13 (September), 221-33.

Hogarth, Robin M. and Hillel J. Einhorn (1992), "Order Effects in Belief Updating: The Belief-Adjustment Model," Cognitive Psychology, 24 (1), 1-55.
Johnson, Michael D. (1989), "The Differential Processing of Product Category and Noncomparable Choice Alternatives," Journal of Consumer Research, 16 (December), 300-309.

Kardes, Frank R. and Gurumurthy Kalyanaram (1992), "Order-ofEntry Effects on Consumer Memory and Judgment: An Information Integration Perspective," Journal of Marketing Research, 29 (August), 343-57.

Mantel, Susan P. and Frank R. Kardes (1999), "The Role of Direction of Comparison, Attribute-Based Processing, and Attitude-Based Processing in Consumer Preference," Journal of Consumer Research, 25 (March), 335-52.

Posavac, Steven S., David M. Sanbonmatsu, Frank R. Kardes, and Gavan J. Fitzsimons (2004), "The Brand Positivity Effect: When Evaluation Confers Preference," Journal of Consumer Research, 31 (December), 643-51.

Russo, J. Edward, Margaret G. Meloy, and Victoria H. Medvec (1998), "Predecisional Distortion of Product Information," Journal of Marketing Research, 35 (November), 438-52.

Russo, J. Edward, Margaret G. Meloy, and T. Jeffrey Wilks (2000), "Predecisional Distortion of Information by Auditors and Salespersons," Management Science, 46 (January), 13-27.

Simon, Dan, Daniel C. Krawczyk, and Keith J. Holyoak (2004), "Construction of Preferences by Constraint Satisfaction," Psychological Science, 15 (5), 331-36. 
Copyright of Journal of Consumer Research is the property of Journal of Consumer Research. Inc. and its content may not be copied or emailed to multiple sites or posted to a listserv without the copyright holder's express written permission. However, users may print, download, or email articles for individual use. 